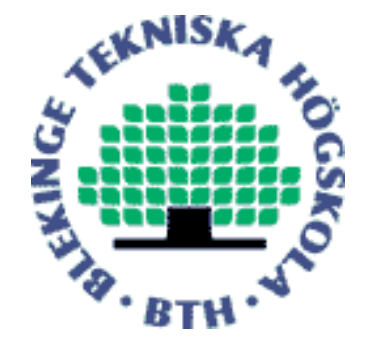

Copyright (C) 2014 IEEE.

Citation for the published paper:

SAR Resolution Enhancement with Circular Aperture in Theory and Empirical Scenario

Viet Thuy Vu, Dheeraj Nehru, Mats Pettersson, Thomas Sjögren

RadarCon

2014 Cincinnati, Ohio

This material is posted here with permission of the IEEE. Such permission of the IEEE does not in any way imply IEEE endorsement of any of BTH's products or services Internal or personal use of this material is permitted. However, permission to reprint/republish this material for advertising or promotional purposes or for creating new collective works for resale or redistribution must be obtained from the IEEE by sending a blank email message to pubs-permissions@iee.org.

By choosing to view this document, you agree to all provisions of the copyright laws protecting it. 


\title{
SAR Resolution Enhancement with Circular Aperture in Theory and Empirical Scenario
}

\author{
Dheeraj N. Nehru*,Viet T. Vu*,Thomas K. Sjögren and Mats I. Pettersson \\ Blekinge Institute of Technology, Karlskrona, 37143 Sweden \\ Email: dheeraj.nehru@ stud.bth.se, viet.thuy.vu@bth.se
}

\begin{abstract}
SAR systems synthesizing circular apertures have been shown to result in better spatial resolutions than the ones synthesizing linear apertures. The paper presents an investigation about the enhancement of SAR spatial resolutions with the use of circular aperture. A comparison between the spatial resolutions obtained with a SAR system synthesizing a circular aperture and with the same SAR system synthesizing a linear aperture is therefore carried out. The studying results are verified by the experimental SAR data set provided by the experimental ground-based SAR system of Blekinge Institute of Technology (BTH GB-SAR).
\end{abstract}

\section{INTRODUCTION}

Synthetic Aperture Radar (SAR) is a radar sensor system which allows high resolution imagery and inherits advantage of usability in any environment because of its operating ability irrespective of severe climatic conditions and daylight. SAR is described as a system which assumes large aperture by synthesizing from the linear movement of radar platform. The requirement for high resolution images is inevitable due to the growing applications of SAR systems. For example, forest vegetation analyses using SAR will be more accurate with the enhanced resolution images [1]. Let it be any kind of monitoring applications such as monitoring monument or landslides or hill slides, utilization of high resolution SAR imagery always yields accurate results [2]. This requirement is the motivations for researchers to develop novel strategies for the resolution enhancement of SAR images.

In order to improve the resolution of SAR images increasing the integration angle and the center frequency of a SAR system and/or using the wider signal bandwidth are the main solutions. Using the wider signal bandwidth for a SAR system can lead to some challenges. For example, that SAR system has a higher possibility to be affected by radio frequency interference (RFI) caused by other radio systems on the ground scene. Increasing the center frequency for a SAR system causes a greater propagation loss and difficulties of generating and amplifying the signal energy. Using a higher integration angle can be a better option but this option depends strongly on the antenna beamwidth utilized by a SAR system. In addition, for a linear aperture, the maximum integration angle is always less than $180^{\circ}$. To increase integration angle, a strategy has been introduced in [3], [4] which suggest the use of circular apertures. This strategy has been shown as a method to enhance SAR resolution.

\footnotetext{
*The two first authors contributed equally to this work.
}

The spatial resolutions, which can be obtained with a SAR system in theory, have been investigated in [5], [6]. For a SAR system synthesizing linear aperture (LSAR), the azimuth resolution is shown to be dependent on the integration angle, the center frequency and the so-called azimuth/range coupling factor in azimuth of that SAR system whereas the range resolution is inversely proportional to the radar signal bandwidth and proportional to the azimuth/range coupling factor in range. The mentioned azimuth/range coupling factors are show to be the functions of fractional bandwidth and integration angle. For a SAR system synthesizing circular aperture (CSAR), the unique spatial resolution depends on the radar signal bandwidth and another factor which is defined by the fractional bandwidth and the incident angle. An investigation into the relationship between the resolutions obtained with LSAR and CSAR or in other words the investigation into the enhancement of SAR spatial resolution with CSAR is necessary.

In this paper, a comparison between the spatial resolutions obtained with LSAR and CSAR in theory and in empirical scenario is presented. Presently, there are not many available SAR systems synthesizing circular apertures (CSAR). Few systems are available like CARABAS [7] which are mainly exploited by the defence agencies and thus SAR data becomes difficult to access. Some CSAR data sets like GOTCHA [8] are available but it is not allowed to mail these data sets out of the country of origin. The comparison between the spatial resolutions obtained with LSAR and CSAR in practice will therefore be relied on the experimental ground-based SAR system of Blekinge Institute of Technology (BTH GBSAR) [9]. The GB-SAR system is quite helpful to carry out research which restrains the unmanageable expenses that arise of carrying out measurement campaigns.

The rest of this paper is organized as follows. Section II presents an investigation into the spatial resolutions obtained with LSAR and CSAR in theory. An investigation into the spatial resolutions obtained with LSAR and CSAR in an empirical scenario is given in Section III. Also in this section, utilizing the BTH GB-SAR system for this investigation and the proposed radar reflector for measurements with circular apertures are presented in detail. The important findings of the study are summarized in the Section IV.

\section{LSAR AND CSAR RESOLUTION IN THEORY}

In this section, an investigation into the spatial resolutions obtained with LSAR and CSAR in theory will be presented. 
The resolutions equations for LSAR and CSAR are therefore utilized for analysis. The verification of the analysis is based on simulated LSAR and CSAR data.

\section{A. LSAR and CSAR resolution equations}

For a LSAR system, the common assessments for resolution are azimuth and range resolutions. The former corresponds to the motion of the platform whereas the later is associated with radar range. In [5], the azimuth resolution in a general case is defined by

$$
\Delta_{\rho_{x}}=\epsilon_{x} \frac{0.2211 \lambda_{c}}{\sin \left(\frac{\phi_{0}}{2}\right)}
$$

where $\lambda_{c}$ is the wavelength of center frequency and $\phi_{0}$ is the effective integration angle, and $\epsilon_{x}$ is a factor showing the azimuth/range coupling. This factor appears mainly in ultrawideband and ultrawidebeam systems whose azimuth/range coupling is serious. For narrowband and narrowbeam system, this factor can be approximated by one. Equation (1) is therefore simplified to

$$
\Delta_{\rho_{x}}=\frac{\lambda_{c}}{4 \sin \left(\frac{\phi_{0}}{2}\right)}
$$

or even

$$
\Delta_{\rho_{x}}=\frac{\lambda_{c}}{2 \phi_{0}}
$$

for very small effective integration angle. From the azimuth equations, it could be understood that azimuth resolution can be enhanced with the use of higher center frequency and/or higher effective integration angle.

The range resolution equation is derived in [5] as a function of radar signal bandwidth $B$ and given by

$$
\Delta_{\rho_{y}}=\epsilon_{y} \frac{0.4422 \mathrm{c}}{B}
$$

where $\mathrm{c}$ represents the speed of propagation and is equal to that of light. The azimuth/range coupling factor $\epsilon_{y}$ in (4) has similar physical meaning of the one given in (1). However, the behavior of $\epsilon_{x}$ and $\epsilon_{y}$ with respect to the fractional bandwidth (ratio of bandwidth to center frequency) and effective integration angle is different. In narrowband and narrowbeam cases, the following approximation is valid

$$
\Delta_{\rho_{y}}=\frac{\mathrm{c}}{2 B}
$$

The range resolution equations imply that the wider bandwidth a LSAR system has, the better range resolution that system can be achieved.

For a LSAR system, the same azimuth and range resolutions are obtained when

$$
\phi_{0}=2 \operatorname{acrsin}\left(\frac{\epsilon_{x}}{\epsilon_{y}} \frac{B_{r}}{2}\right) \approx 2 \operatorname{acrsin}\left(\frac{B_{r}}{2}\right)
$$

where $B_{r}$ denotes fractional bandwidth.

For a CSAR system, since the trajectory of SAR platform is circular path, azimuth and range directions do not exist and thus there is only one unique spatial resolution which

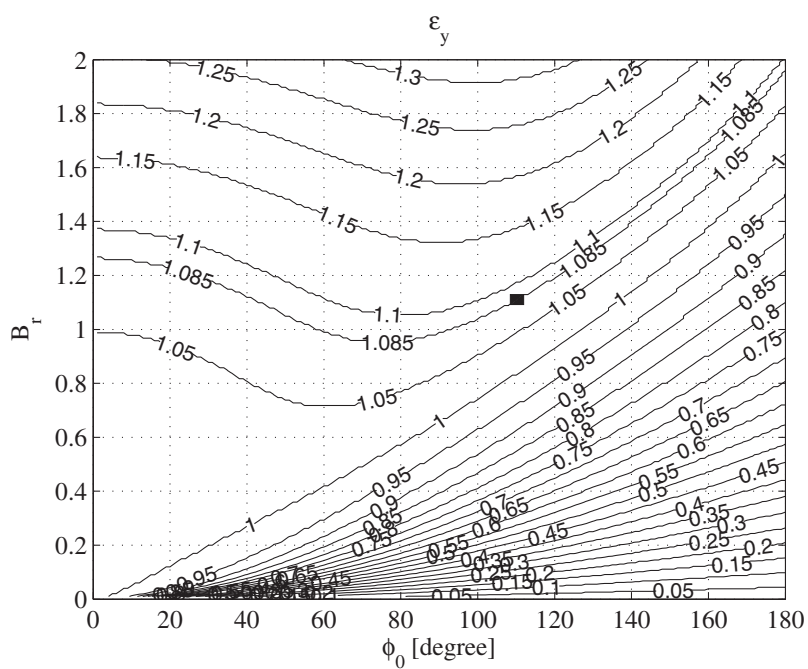

Fig. 1. Azimuth/range coupling factor $\epsilon_{y}$.

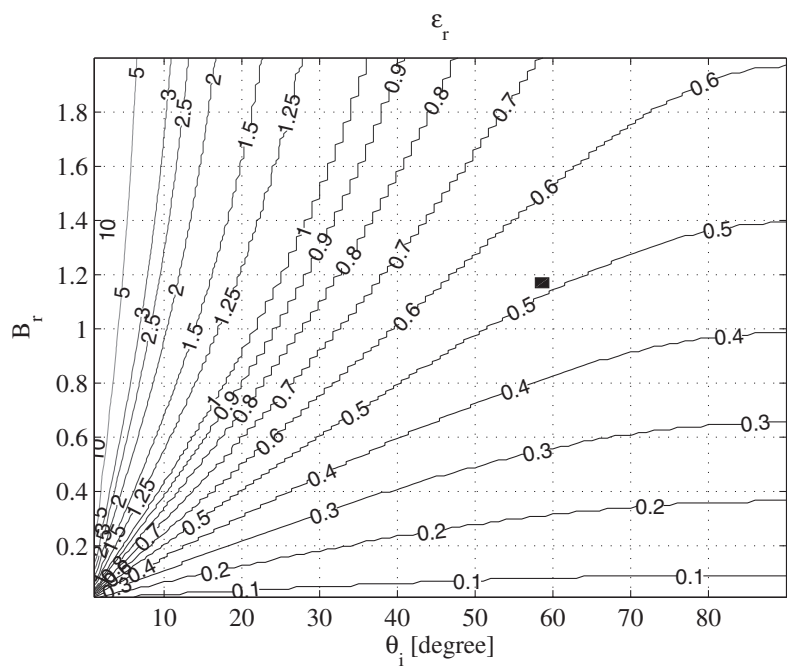

Fig. 2. Broadening/narrowing factor $\epsilon_{r}$.

depends strongly upon the bandwidth. In [6], this dependence is represented by

$$
\Delta_{\rho_{r}}=\epsilon_{r} \frac{0.4422 \mathrm{c}}{B}
$$

where $\epsilon_{r}$ is the broadening/narrowing factor which is show to be a function of incident angle and fractional bandwidth.

\section{B. Analysis}

The investigation into the theoretical LSAR and CSAR resolutions in this paper is limited by range resolution for LSAR and spatial resolution for CSAR. Azimuth resolution is assumed to be equal to range resolution for LSAR. Thus, if we compare (4) and (7), we can see that the difference between the range resolution of a LSAR system and spatial resolution of a CSAR system is decided by the azimuth/range coupling factor $\epsilon_{y}$ and the broadening/narrowing factor $\epsilon_{r}$. Fig. 1 and Fig. 2 
TABLE I

PARAMETERS FOR LSAR AND CSAR SIMULATIONS.

\begin{tabular}{ccc}
\hline \hline Parameter & LSAR & CSAR \\
\hline The highest processing frequency & $2.5 \mathrm{GHz}$ & $2.5 \mathrm{GHz}$ \\
The lowest processing frequency & $1.5 \mathrm{GHz}$ & $1.5 \mathrm{GHz}$ \\
Altitude of the ground-based SAR system & $1.25 \mathrm{~m}$ & $1.25 \mathrm{~m}$ \\
Minimum range & $2.86 \mathrm{~m}$ & $2.86 \mathrm{~m}$ \\
Aperture step & $0.05 \mathrm{~m}$ & $0.10 \mathrm{~m}$ \\
Synthetic aperture length & $1.2 \mathrm{~m}$ & $16 \mathrm{~m}$ \\
Number of aperture positions & 25 & 150 \\
\hline \hline
\end{tabular}

show the behavior of these factors with respect to fractional bandwidth and integration angle or incident angle.

As observed in Fig. 1 and Fig. 2, for SAR systems with conventional parameters, CSAR system results in much better resolution than LSAR systems. For example, for CARABAS with the fractional bandwidth of about 1.16, the integration angle of $110^{\circ}$, and the incident angle of $58^{\circ}$ (marked by the solid black rectangles in Fig. 1 and Fig. 2), the factors are approximately 1.085 for LSAR and 0.5 for CSAR. This means the resolution can be enhanced more than two times if CARABAS synthesizes a circular aperture instead of a linear aperture.

However, if we only the increase integration angle and keep the fractional bandwidth low for a LSAR system (lower right corner of Fig. 1), the coupling factor can be reduced significantly, i.e. the SAR resolution is enhanced. It should be observed that even though this change reduces the coupling factor, such case can lead to narrow bandwidth signal which again may not enhance the resolution because of the presence of signal bandwidth in the denominator of range resolution equation (3). The same effect can be obtained with a CSAR system by increase in incident angle and decrease in fractional bandwidth (lower right corner of Fig. 2). In these cases, the enhancement in resolution of CSAR is not significantly in comparison to LSAR. It should be noticed that for high incident angles, either the radius of circular aperture may be too large or the altitude of the radar platform may be too low.

In contrary, if we only the increase fractional bandwidth and keep the integration angle low for a LSAR system (upper left corner of Fig. 1), this will not help to enhance the range resolution. The resolution will be degraded (up to 30\%) if the LSAR system utilizes too high fraction bandwidth and too low integration angle. The same situation happens for a CSAR system with high fractional bandwidth and low incident angle (upper left corner of Fig. 2). However, the degradation in this case is extremely serious (even more than 10 times).

\section{LSAR and CSAR simulations}

In this subsection, we present some simulation results for illustrating the analysis in the previous subsection. We select randomly a set of SAR parameters to simulate a LSAR system and a CSAR system illuminating a ground scene. The SAR scene includes only a single point-like scatterer located in

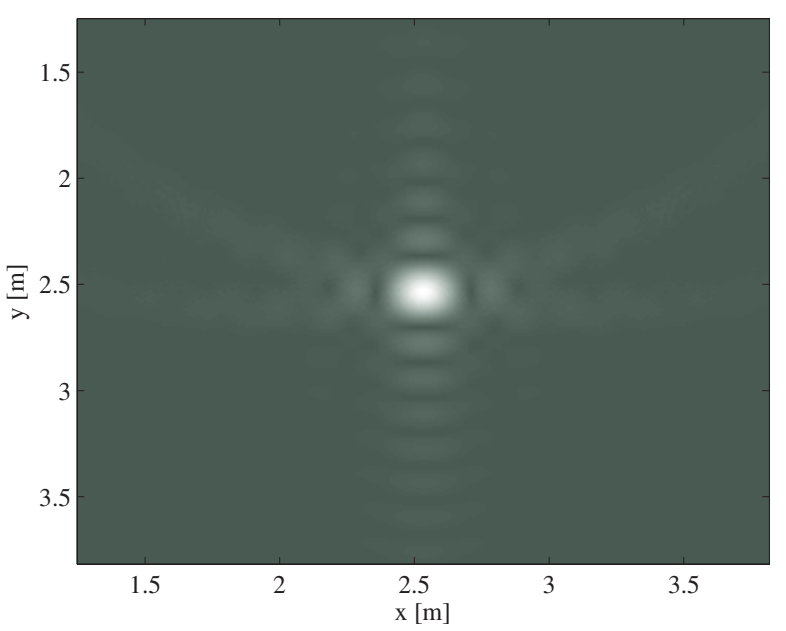

Fig. 3. SAR image formed with simulated LSAR data.

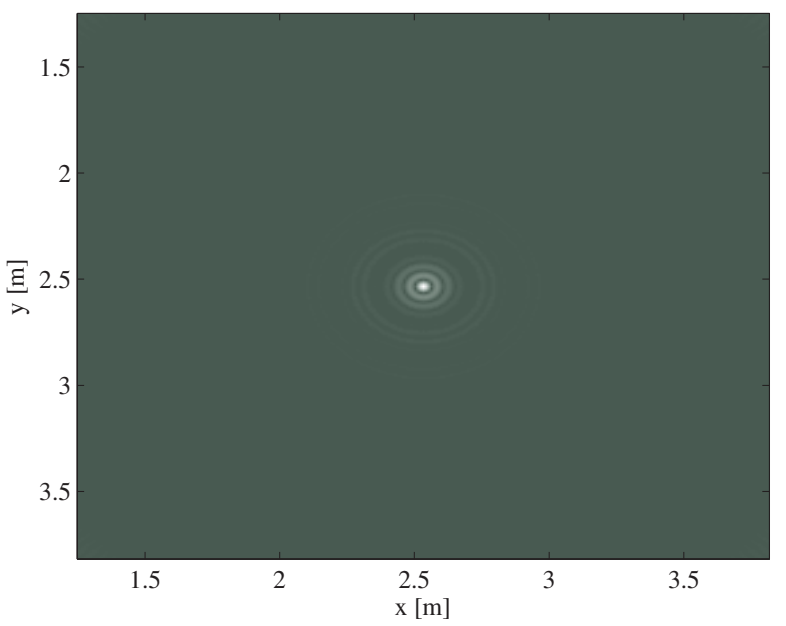

Fig. 4. SAR image formed with simulated CSAR data.

the middle of the scene. The simulated data is exploited to reconstruct the SAR scene. The resolutions of the SAR images are measured and evaluated. For convenience, we select the parameters of the BTH GB-SAR system and these parameters are summarized in Table I.

For the LSAR simulation, the azimuth and range resolutions are expected to be the same due to the limitation of the investigations. The integration angle being synthesized by the system is therefore estimated from (6) with the consideration of the coupling and broadening/narrowing factors. The estimation gives the integration angle of $48^{\circ}$. Using the condition for aperture step size derived in [9], we can show the aperture step size to be $0.05 \mathrm{~m}$. In the LSAR simulation, the spotlight mode is considered. In this mode, the antenna is rotated in order to keep itself in a direct contact with the scatterer during movement of the antenna. In other words, the antenna is always in contact with the scatterer through its mainlobe. This 


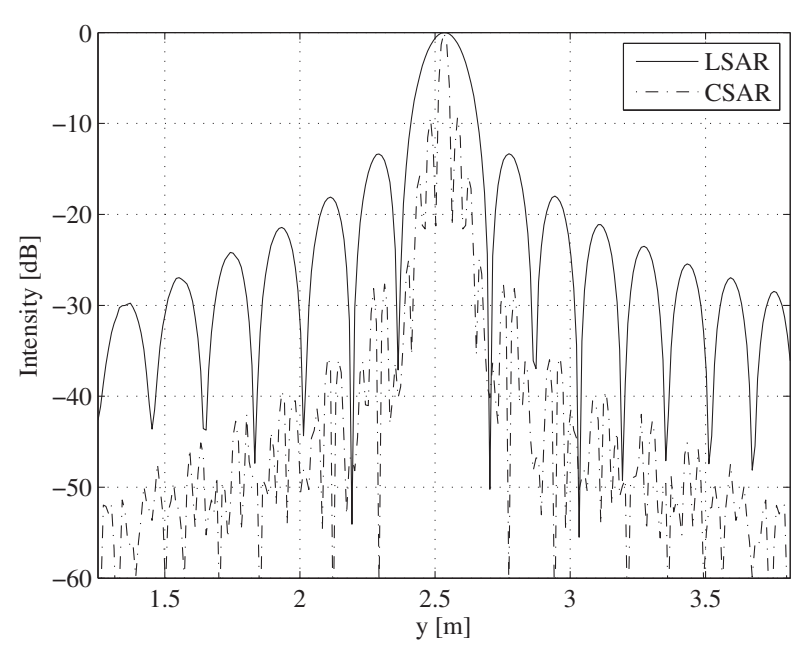

Fig. 5. Vectors extracted from images formed with simulated data in $y$ direction.

TABLE II

MEASUREMENT RESULTS

\begin{tabular}{ccc}
\hline \hline Parameter & LSAR & CSAR \\
\hline Resolution in theory & $\approx 0.14 \mathrm{~m}$ & $\approx 0.03 \mathrm{~m}$ \\
Resolution in empirical scenario & $\approx 0.15 \mathrm{~m}$ & $\approx 0.03 \mathrm{~m}$ \\
\hline \hline
\end{tabular}

results in a swath only on the SAR scene during the entire path of motion. Fig. 3 shows the SAR image reconstructed with the simulated data using the Global Backprojection (GBP) algorithm [10]. As shown, the point-like scatterer is well focused and appears as a point target. The characteristics of wide signal bandwidth and wide integration angle can be clearly observed from Fig. 3 (non- and orthogonal sidelobes).

For the CSAR simulation, we use the same parameters. The incident angle is easily calculated as $64^{\circ}$ by applying trigonometric function for the altitude and the minimum range. If we use the same aperture step size, i.e. $0.05 \mathrm{~m}$, the number of aperture positions will be 320 , i.e. more than 12 times number of aperture positions in the LSAR case. The aperture length is up to $16 \mathrm{~m}$. If we use the same number aperture positions, i.e. 25 , the aperture step increases to $0.64 \mathrm{~m}$. Our other simulations show that the aperture step does not affect the spatial resolution of CSAR. The major effect caused by big aperture steps, i.e. unwanted high sidelobe level, does not appear if we double the aperture step, i.e. $0.1 \mathrm{~m}$. The number of aperture positions is therefore increased to 150 . The SAR image formed with these calculated values is given in Fig. 4. One can see directly that the resolution of the image formed with the CSAR data is enhanced significantly in comparison to the one formed with the LSAR data given in Fig. 3. For a better evaluation of this enhancement, the vectors in the y direction are extracted and then the resolution measurements are performed on these vectors. The extracted vectors are plotted in Fig. 5 showing again the enhancement

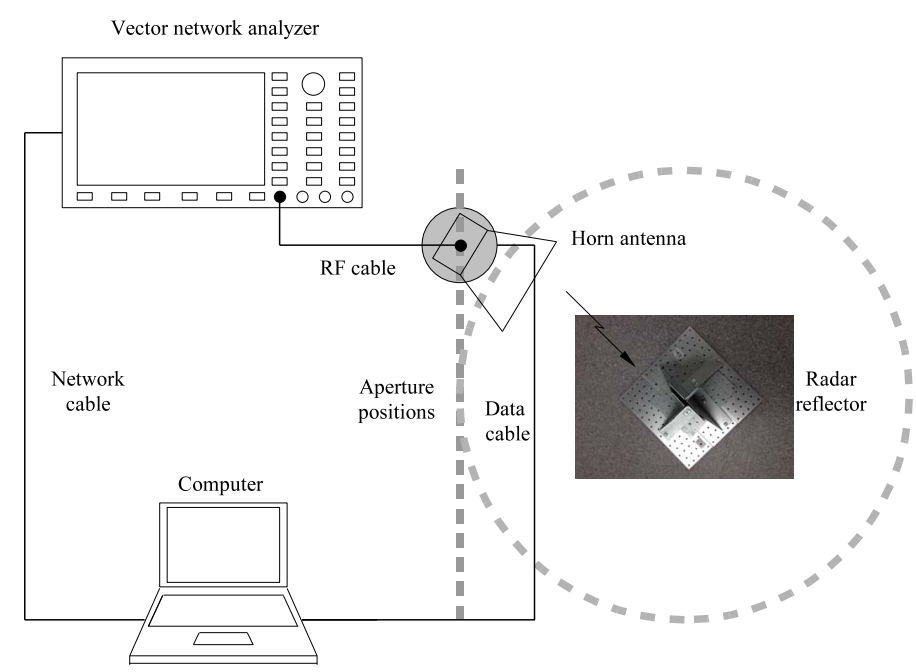

Fig. 6. BTH GB-SAR system diagram.

in resolution of CSAR. In Table II, we provide the values of resolution measured on the extracted vectors which are quite matched with the values estimated with the resolution equations. The results indicate that the circular aperture offers a much better resolution in comparison to the linear aperture. The improvement in resolution with CSAR in this case can be expressed by a factor of about 5 .

\section{LSAR AND CSAR RESOLUTION IN EMPIRICAL SCENARIO}

Spatial resolution enhanced with CSAR in an empirical scenario will be investigated in this section. The investigation is relied on the BTH GB-SAR system. The LSAR and CSAR data is acquired with this system and then processed according to the investigation purposes.

\section{A. BTH GB-SAR and radar reflector}

BTH GB-SAR is a flexible ground-based system [9]. The flexibility is found from the wide operating frequency range of the system, the unlimited length of the synthetic aperture, the features of the synthetic aperture, e.g. linear and circular, and different acquisition modes of the system, e.g. stripmap and spotlight.

Fig. 6 shows the diagram of this system. The main components of the system include a vector network analyzer, a double ridge guide horn antenna, a computer, cables, antenna positioning, and other accessories.

It is common knowledge that a normal dihedral corner reflector can only reflect incident wave from certain directions and therefore not appropriate for measurements with a circular aperture. We propose to use a special radar reflector having diamond shape in the following experiments. The structure of this radar reflector allows reflecting incident wave from any direction. The maximum dimension of the radar reflector is about $50 \mathrm{~cm}$. Although the dimension of the radar reflector is not optimum for the selected frequency range its reflection is still significant. 


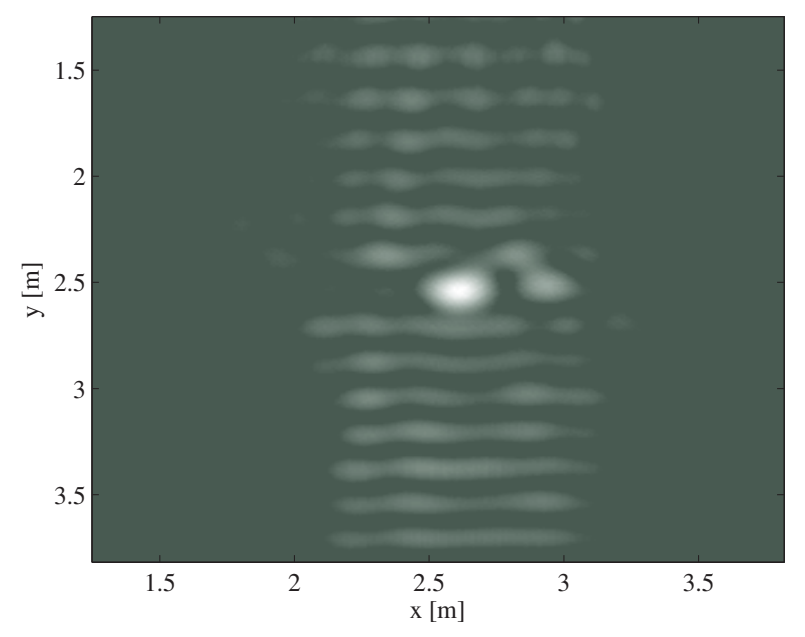

Fig. 7. SAR image formed with real LSAR data.

\section{B. Experiments}

The BTH GB-SAR system is configured according to the parameters given in Table I. The SAR scene is the radio communication laboratory of BTH. The diamond shaped radar reflector is located in the center of the SAR scene.

The operation mode of the system in data acquisition is spotlight. This mode of operation helps during the experimentation in an environment like laboratory to obtain a maximum reflection from the reflector which otherwise contains reflections from other apparatus in the laboratory.

The system will collect the data for the LSAR and CSAR cases. Again, all the parameters used of LSAR are same to that of CSAR apart from the aperture steps and the numbers of aperture positions. The aperture steps and the numbers of aperture positions are identical to the ones which have been used for the LSAR and CSAR simulations. The procedure of data acquisition is vividly portrayed in the [9]. It should be noticed that we should use a small window for the gating function in the vector network analyzer to suppress as much as possible the reflections from other apparatus in the laboratory, especially in the CSAR case where the radar range with respect to the radar reflector is constant.

\section{Measurements}

The LSAR and CSAR data are used to reconstruct the SAR scene using the GBP algorithm. Fig. 7 and Fig. 8 provide the images of the SAR scene illuminated by the GB-SAR system synthesizing linear and circular aperture, respectively. The radar reflector appears in Fig. 7 as a point-like scatterer whereas the detail of the radar reflector can be observed in Fig. 8. The structure of the radar reflector given in the SAR image in Fig. 8 is similar to the structure in reality which can see from Fig. 6. This due to the significant improvement in resolution of the CSAR case.

Fig. 9 shows the vectors extracted from Fig. 7 and Fig. 8 in the $y$ direction for resolution measurements. The measurement

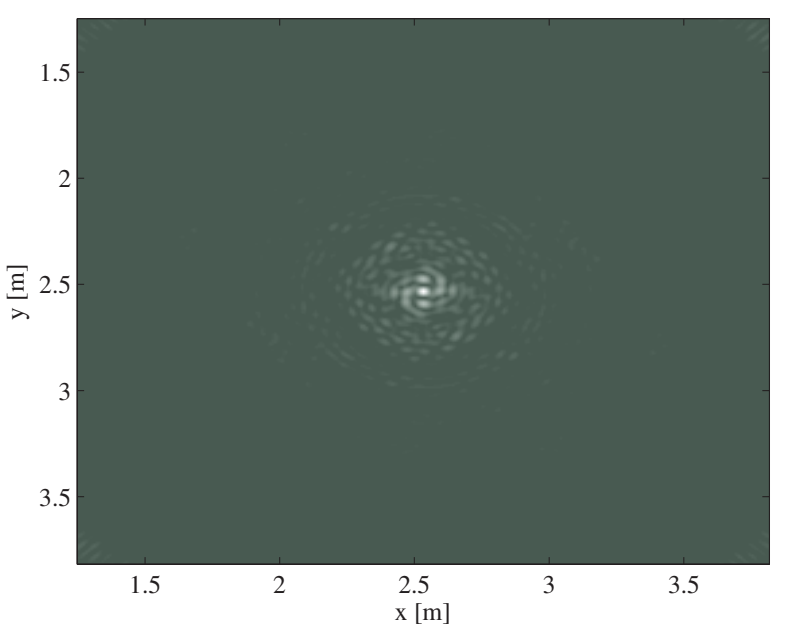

Fig. 8. SAR image formed with real CSAR data.

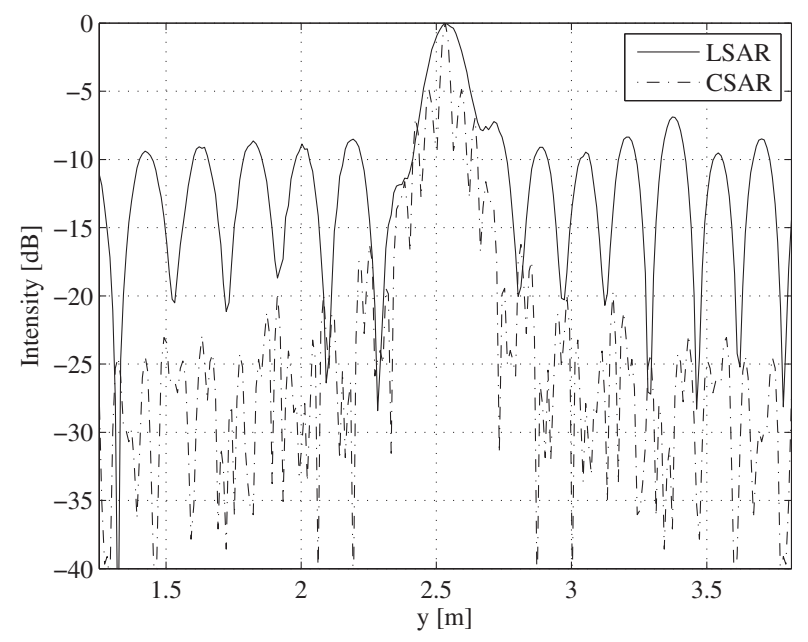

Fig. 9. Vectors extracted from images formed with real data in $y$ direction.

results given in Table II indicate that the resolutions in theory and in the empirical scenario are almost identical. In other words, the enhancement in resolution of CSAR in practice is similar to that in theory. The improvement in resolution with CSAR in this case can also be expressed by a factor of about 5 .

\section{CONCLUSION}

This paper deals with the strategy to increase resolution of SAR image with circular aperture. The effects with the use of circular aperture are vividly explained by the theoretical analysis followed by the simulated results and the practical observations. In order to restrain from the unmanageable expenses of carrying out field campaigns, the empirical data is obtained with the BTH GB-SAR system. The unique structure of the radar reflector has been used to achieve prominent reflections while collecting the practical data using linear and 
circular apertures. The enhancement of resolution is evident from the results obtained and the resolution achieved by CSAR is around 5 times better than that of LSAR ones with the chosen parameter set. However, there is a mismatch between the theoretical and practical results which could be an effect due to prorogation loses or caused by the laboratory environment.

\section{ACKNOWLEDGMENT}

The authors would like to thank the KK-Foundation for making this research project possible, and are also appreciative of support from the Swedish Defence Research Agency, Saab Bofors Dynamics, Saab Microwave Systems and RUAG Space.

\section{REFERENCES}

[1] P. Patel, H. S. Srivastava and R. R. Navalgund, "Use of synthetic aperture radar polarimetry to characterize wetland targets of Keoladeo national park, Bharatpur, India", Current Science, vol. 97, no. 4, pp. 529-537, 2009.

[2] N. Casagli, P. Farina, D. Leva, G. Nico and D. Tarchi, "Monitoring the Tessina landslide by a ground-based SAR interferometer and assessment of the system accuracy", in Proc. IEEE IGARSS'2002, vol. 5, Toronto, Canada, Jul. 2002, pp. 2915-2917.

[3] K. Leilei, W. Xiaoqing, Z. Minhui, C. Jinsong and X. Maosheng, "Resolution analysis of circular SAR with partial circular aperture measurements", in EUSAR'2010, Aachen, Germany, pp. 1-4, 2010.

[4] R. Sharma, "Analysis of circular aperture SAR image formation", in Forty-Third Asilomar Conference on Signals, Systems and Computers, Pacific Grove, CA, 2009, pp. 482-487.

[5] V. T. Vu, T. K. Sjögren and M.I. Pettersson, "On synthetic aperture radar azimuth and range resolution equations", IEEE Trans. Aerosp. Electron. Syst., vol. 48, no. 2, pp. 1764-1769, 2012.

[6] V. T. Vu, T. K. Sjögren and M.I. Pettersson, "Studying CSAR systems using IRF-CSAR", in Proc. IET RADAR, Glasgow, UK, Oct. 2012, pp. $1-6$.

[7] P.-O. Frölind, A. Gustavsson, M. Lundberg and L.M.H Ulander, "Circular-aperture VHF-band synthetic aperture radar for detection of vehicles in forest concealment," IEEE Trans. Geosci. Remote Sensing, vol. 50, no. 4, pp. 1329-1339, 2012.

[8] E. Ertin, C. D. Austin, S. Sharma, R. L. Moses and L. C. Potter "GOTCHA experience report: Three-dimensional SAR imaging with complete circular apertures," SPIE Algorithms for Synthetic Aperture Radar Imagery XIV, vol. 6568, Orlando, FL, Apr. 2007. pp. 02-13.

[9] V. T. Vu, D. N. Nehru, T. K. Sjögren and M.I. Pettersson, "An experimental ground-based SAR system for studying SAR fundamentals", in Proc. APSAR 2013, Tsukuba, Japan, Sep. 2013, pp. 424-427.

[10] L. E. Andersson, "On the determination of a function from spherical averages", SIAM Journal on Mathematical Analysis, vol. 19, no. 1, pp. 214-232, 1988 